\title{
DESENVOLVIMENTO DO JOGO NOVA LUZ PARA UTILIZAÇÃO NA PSICOTERAPIA EM CASOS DE TRANSTORNOS ALIMENTARES
}

\author{
DESARROLLO DEL JUEGO NOVA LUZ PARA USO EN PSICOTERAPIA EN CASOS \\ DE TRASTORNOS ALIMENTARIOS
}

\section{DEVELOPMENT OF THE NOVA LUZ GAME FOR USE IN PSYCHOTHERAPY IN CASES OF FOOD DISORDERS}

\author{
Raquel SARTORATO ${ }^{1}$ \\ Luísa Pelucio RIBEIRO ${ }^{2}$
}

RESUMO: A abordagem cognitivo-comportamental se baseia no modelo cognitivo associado às técnicas e estratégias específicas, visando à modificação de pensamentos, crenças e comportamentos disfuncionais, relacionados ao sofrimento ou transtorno emocional do paciente. Embora a TCC tenha se tornado aceita como um dos principais métodos de tratamento para transtornos alimentares, estudos ainda estão em crescimento no Brasil. O presente artigo propõe o desenvolvimento do jogo Nova Luz como recurso para ser utilizado em psicoterapia nos casos de TA. A construção foi feita de acordo com a literatura já existente e o conhecimento adquirido. Assim, alcançou o propósito de criar um artificio de forma que promovesse momentos de psicoeducação e contribuísse para a averiguação de fatores importantes para o processo psicoterapêutico, de maneira menos tensa e mais espontânea durante a sessão, quando o paciente traz a queixa relacionada a questão alimentar.

PALAVRAS-CHAVE: Terapia cognitivo-comportamental. Transtornos alimentares. Jogo.

RESUMEN: El enfoque cognitivo-conductual se basa en el modelo cognitivo asociado a técnicas y estrategias específicas, con miras a la modificación de pensamientos, creencias y comportamientos disfuncionales, relacionados con el sufrimiento o trastorno emocional del paciente. Aunque la TCC se ha aceptado como uno de los principales métodos de tratamiento para los trastornos alimentarios, los estudios aún se están desarrollando en Brasil. Este artículo propone el desarrollo del juego Nova Luz como recurso para ser utilizado en psicoterapia en casos de TA. La construcción se realizó acorde con la literatura existente y los conocimientos adquiridos. Así, logró el propósito de crear un artificio de manera que propiciara momentos de psicoeducación y contribuyese a la investigación de factores importantes para el proceso psicoterapéutico, de forma menos tensa y más espontánea durante la sesión, cuando el paciente trae la queja relacionada con el tema alimentario.

PALABRAS CLAVE: Terapia de cognitivo-conductuales. Trastornos de la alimentación. Juego.

\footnotetext{
${ }^{1}$ Centro Universitário de Barra Mansa (UBM), Barra Mansa - RJ - Brasil. Graduada no curso de Psicologia. ORCID: https://orcid.org/0000-0002-5940-4304. E-mail: raquelsartorato@ hotmail.com

${ }^{2}$ Universidade Estácio de Sá (UNESA), Resende - RJ - Brasil. Coordenadora da Pós Graduação em Terapia Cognitivo Comportamental - UBM. Mestrado em Psiquiatria e Saúde Mental (UFRJ). ORCID: https://orcid.org/0000-0002-7928-2578. E-mail: luisapelucio@hotmail.com
} 
ABSTRACT: The cognitive-behavioral approach is based on the cognitive model associated to specific techniques and strategies, aiming at modifying thoughts, beliefs and dysfunctional behaviors related to the patient's suffering or emotional disorder. Although CBT has become accepted as one of the main methods of treatment for eating disorders, studies are still growing in Brazil. This article proposes the development of the Nova Luz game as a resource to be used in psychotherapy in cases of Eating Disorder. The construction was done according to the existing literature and the acquired knowledge. Thus, it achieved the purpose of creating an artifice that would promote moments of psychoeducation and contribute to the investigation of important factors for the psychotherapeutic process, in a less tense and more spontaneous way during the session, when the patient brings the complaint related to food issues.

KEYWORDS: Cognitive behavioral therapy. Eating disorders. Game.

\section{Introdução}

Atualmente, a cultura alimentar se caracteriza pelo enaltecimento da grande quantidade de comida, desde que com baixo custo e preferencialmente em pouco espaço de tempo. Assim, rodízios são vangloriados, tendo como objetivo o lucro pessoal, comendo-se em grande quantidade, servido a todo o tempo e utilizando do benefício do custo "em conta". Prosseguindo nessa ideia de custo, existem também os combos e promoções, que se expandiram com o advento dos aplicativos de celular, com alguns ofertando o serviço de delivery, agradando muitos usuários devido a comodidade.

Logo, de acordo com a lei da oferta e da procura, a tendência é que esse comportamento, da valorização da comida em grande quantidade e o preço, continue se incorporando em meio a sociedade. Em contrapartida, existe um padrão de beleza ditado ao coletivo que exalta o corpo magro, novamente destacado ainda mais pela tecnologia e redes sociais, representando autodisciplina. Assim, pode-se perceber a contradição e a pressão social que envolve a percepção da imagem e comportamentos acerca da alimentação (OLIVEIRA; HUTZ, 2010).

Uma possibilidade de vivenciar o prazer é por meio da comida, por vezes ultrapassando o nível de comer para se alimentar, podendo ser exemplificado claramente na compulsão alimentar, que está atrelada ao descontrole momentâneo, se rendendo, seja ao puro prazer de comer ou para mascarar sentimentos e experiências ruins. Entretando, a culpa aparecendo em seguida, pode levar a comportamentos compensatórios, relacionados a um quadro bulímico (BORGES et al., 2006). A comida também pode ser vista como vilã, optando 
por evitá-la a todo e qualquer custo, pois ela impede de chegar ao que se julga ser o corpo ideal, no caso da anorexia, um ideal inatingível.

Vivemos em uma época de exposição, mais uma vez com relação à modernidade e tecnologia. As redes sociais favorecem a comparação com o próximo, nesse mundo, o que importa é o visual, a imagem, a vaidade, a quantidade de seguidores e curtidas. O que importa na realidade é ser aceito. Aceito mesmo que por desconhecidos, mesmo que seja com o recurso de photoshop, para que se aparente mais magro, com a barriga mais definida, rosto afinado, glúteos e seios maiores, pois é assim o corpo idealizado, e a vontade de ser dessa maneira aumenta dentre vários usuários. A mídia, em grande parte, reconhece e reforça tal comportamento.

Complicações médicas podem surgir em decorrência da desnutrição e dos comportamentos purgativos compensatórios, tais como anemia, alterações endócrinas, osteoporose e alterações hidroeletrolíticas (especialmente hipocalemia, que pode levar a arritmia cardíaca e morte súbita), entre outras. Já as complicações clínicas são decorrentes principalmente das manobras compensatórias para perda de peso, como: erosão dos dentes, alargamento das parótidas, esofagites, hipopotassemia e alterações cardiovasculares, outras (LEAL; CABRAL, 2002).

Sobre o histórico do interesse da psicologia frente aos transtornos alimentares, se deu na decada de 40 (OLIVEIRA; DEIRO, 2013), após isso, outras abordagens foram se destacando também devido a divergências de concepções sobre o conduzir do assunto. Ainda assim, autores defendem que são necessários mais estudos acerca do assunto.

Pode ser observado que pessoas acometidas por transtornos alimentares normalmente sentem uma profunda culpa por estarem em tal situação, por isso, é frequente que o ciclo social demore a perceber os sintomas e que essa pessoa procure voluntariamente ajuda profissional.

Uma vez no consultório, paira o assunto delicado, carregado por sentimento de vergonha, entretanto, é fundamental avaliar os sintomas, pensamentos e comportamentos do paciente. Para que essa avaliação seja feita de maneira menos conservadora, o brincar pode ser uma solução, em virtude de que essa estratégia conecta o mundo exterior com o interior como defende Affonso (2012), sendo muito utilizado como estratégias em psicoterapias infantis, incluindo na abordagem cognitivo-comportamental na qual o trabalho se baseia. Entretanto, propõe-se que o lúdico seja experimentado para outras fases do desenvolvimento, de modo que possa ser ilustrado da melhor maneira a queixa trazida pelo paciente referente ao transtorno alimentar (TA). 
Por conseguinte, esse artigo tem por objetivo abordar a queixa do transtorno alimentar, encontrada nos consultórios de psicoterapia, bem como as estratégias cognitivas comportamentais utilizadas, norteando o desenvolvimento do jogo "Nova Luz". Elaborado intencionalmente para a utilização em sessões de psicoterapia, tendo como proposta a avaliação de sintomas, pensamentos e comportamentos disfuncionais, discorrendo sobre a autoestima, favorecendo a relação paciente-terapeuta e promovendo momentos de psicoeducação.

Para isso, será apresentado, inicialmente, um breve histórico dos transtornos alimentares, recaptulando os primeiros registros encontrados sobre a incidência destes, as definições e sintomas de anorexia, bulimia e compulsão alimentar, envolvendo a etiologia multifatorial e dissertando sobre o percurso da TCC relacionado ao tema transtorno alimentar.

\section{Transtornos Alimentares}

Conforme a American Psychiatric Association, os transtornos alimentares são compreendidos pela disfunção do sujeito, relacionada ao alimento, em intensidade, frequência e comportamentos resultantes dessa relação, havendo prejuízo à saúde física e ao funcionamento psicossocial. São compreendidos por: Anorexia, Bulimia, Compulsão Alimentar, Pica e Transtorno Restritivo/ Evitativo, enquanto os três primeiros citados apresentam uma preocupação com o corpo, com a magreza e a disformia corporal, motivando os esforços e comportamentos disfuncionais (DUCHESNE; ALMEIDA, 2002).

A palavra anorexia vem do grego A (negativo, sem) + OREXIS (apetite, desejo) significando sem apetite. Apesar dos estudos terem se iniciado por volta do século XVII, os casos relacionados aos sintomas dos transtornos alimentares já se apresentavam séculos antes.

No século III, por exemplo, alegam que existiam relatos médicos de pacientes acometidos por o que se assemelhava a anorexia, como o caso de um Buda que buscava iluminação através da inanição. Logo, a anorexia teve reconhecimento pela primeira vez na Idade Média, como uma causa misteriosa que tendia a uma dieta de fome autoimposta com significativa perda de peso (OLIVEIRA, 2004). Assim, durante a Idade Média, as práticas de jejum foram compreendidas como estados de possessão demoníaca ou milagres divinos. Cordás (2004) afirma que "No século XIII, encontrou-se em grande profusão descrições de mulheres que se autoimpunham jejum como uma forma de se aproximar espiritualmente de Deus; eram as chamadas santas anoréxicas". 
$\mathrm{O}$ primeiro registro de caso se aproximava à anorexia nervosa (AN), foi feito por Richard Morton, no ano de 1689. O autor ainda comenta em seu livro "Tisiologia sobre a doença da consunção" a respeito da influência mútua entre processos mentais e físicos e ressalta o papel patogênico das emoções, ou seja, psicossomático (CORDÁS, 2004) Em um momento próximo, o psiquiatra francês Lasègu, publicou o artigo "De l'anorexie hystérique", acreditando que a etiologia do transtorno era histérica enfatizando as distorções cognitivas, a negação, os transtornos de imagem corporal e o envolvimento familiar (CORDÁS; CLAUDINO, 2002).

Em concordância com a American Psychiatric Association, a anorexia nervosa consiste na restrição da ingesta calórica em relação às necessidades, levando a um peso corporal significativamente baixo no contexto de idade, gênero, trajetória do desenvolvimento e saúde física do indivíduo, devido ao medo intenso de ganhar peso ou de engordar, mesmo estando com peso significativamente baixo. Havendo perturbação no modo como o próprio peso ou a forma corporal são vivenciados, influência indevida do peso ou da forma corporal na autoavaliação ou ausência persistente de reconhecimento da gravidade do baixo peso corporal atual.

Consoante a Cordás e Claudino (2002), o comportamento de forçar o vômito é antigo, podendo ser encontrado precocemente na história de diferentes povos da Antiguidade. No antigo Egito, grande parte do papiro de Eber é dedicado ao estímulo e às virtudes do ato de vomitar. Heródoto acreditava que os egípcios vomitavam e usavam purgativos todo mês, por três dias consecutivos, baseado no julgamento que "todas as doenças dos homens são oriundas da comida". Já na medicina grega, Hipócrates também recomendava a indução de vômitos por dois dias consecutivos todo mês, como um método de prevenir diferentes doenças. Inclusive, os romanos criaram o "vomitorium", que lhes permitia alimentar-se em excesso durante os banquetes, e posteriormente vomitar em local reservado para esta finalidade, às vezes usando uma pena de ave para estimular o reflexo do vômito na garganta. Purgantes eram populares já na Idade Média, junto aos eméticos (medicamentos para induzir o vômito) dominaram o arsenal terapêutico por muitos anos.

Contudo, a primeira descrição da bulimia nervosa foi feita em 1979 pelo psiquiatra inglês Gerald Russel. O termo bulimia tem uma história muito antiga; deriva do grego BOUS (boi) + LIMOS (fome), designando assim um apetite tão grande que seria possível a um homem comer um boi, ou quase (CORDÁS; CLAUDINO, 2002).

Conforme a American Psychiatric Association (2014), o quadro de bulimia nervosa (BN) é caracterizado por episódios recorrentes de compulsão alimentar, que consitem em: 
1. Ingestão, em um período de tempo determinado (por exemplo, dentro de cada período de duas horas), de uma quantidade de alimento definitivamente maior do que a maioria dos indivíduos consumiria no mesmo período sob circunstâncias semelhantes. 2. Sensação de falta de controle sobre a ingestão durante o episódio. Com a presença de Comportamentos compensatórios inapropriados recorrentes a fim de impedir o ganho de peso, como vômitos autoinduzidos, uso indevido de laxantes, diuréticos ou outros medicamentos, jejum ou exercício em excesso. Ocorrendo em média, no mínimo uma vez por semana durante três meses. Sendo a autoavaliação indevidamente influenciada pela forma e pelo peso corporais.

Já a Compulsão Alimentar, qualifica-se por:

1. Ingestão, em um período determinado de uma quantidade de alimento definitivamente maior do que a maioria das pessoas consumiria no mesmo período sob circunstâncias semelhantes. 2. Sensação de falta de controle sobre a ingestão durante o episódio (por exemplo., sentimento de não conseguir parar de comer ou controlar o que e o quanto se está ingerindo). Os episódios de compulsão alimentar estão associados a três (ou mais) dos seguintes aspectos: Comer mais rapidamente do que o normal; Comer até se sentir desconfortavelmente cheio; Comer grandes quantidades de alimento na ausência da sensação física de fome; Comer sozinho por vergonha do quanto se está comendo; Sentir-se desgostoso de si mesmo, deprimido ou muito culpado em seguida. Apresenta um sofrimento marcante em virtude da compulsão alimentar (AMERICAN PSYCHIATRIC ASSOCIATION, 2014).

É importante grifar que a compulsão alimentar não está associada ao uso recorrente de comportamento compensatório inapropriado como na bulimia nervosa e não ocorre exclusivamente durante o curso de bulimia nervosa ou anorexia nervosa. Ademais, o transtorno de compulsão alimentar ocorre em indivíduos de peso normal ou com sobrepeso e obesos. Sendo consistentemente associado ao sobrepeso e à obesidade em indivíduos que buscam tratamento. Contudo, é distinto da obesidade. (AMERICAN PSYCHIATRIC ASSOCIATION, 2014).

É importante frisar, assim como evidenciam Faria e Shinoara (1998), que a obesidade está contida no International Classification of Diseases (ICD) porém não aparece no The Diagnostic and Statistical Manual of Mental Disorders (DSM) como um transtorno alimentar.

\section{Terapia Cognitivo-Comportamental}

A clínica dos transtornos alimentares passou por muitas mudanças durante as últimas décadas. Inicialmente, tinha-se como as principais intevenções o repouso acamado, a alimentação nasogástrica e uma variedade de farmacoterapias, dirigidas principalmente para a recuperação do peso (OLIVEIRA; DEIRO, 2013). 
A psicanálise passou a dominar o entendimento e tratamento a partir dos anos 40 (OLIVEIRA, 2004) e nas décadas seguintes, um regime baseado no reforço para o ganho de peso tornou-se popular com o aparecimento dos programas de tratamento comportamental. (OLIVEIRA; DEIRO, 2013), sendo, entretanto, efetivos a curto prazo, porém, com uma alta taxa de recaída. As terapias cognitivas se destacaram também devido a insatisfação do modelo estritamente comportamental. Assim, a TCC tornou-se aceita como uma das principais abordagens para o tratamento dos transtornos alimentares (DUCHESNE; ALMEIDA, 2002). Entretanto, autores ainda defendem a necessidade de maus estudos contemplando essas areas.

A abordagem cognitivo-comportamental se baseia no modelo cognitivo e na utilização de técnicas específicas, predominantemente cognitivas e comportamentais, que visam à modificação dos padrões de pensamentos e crenças disfuncionais que causam ou mantêm sofrimento emocional e/ou distúrbios psicológicos no indivíduo. Fundamenta-se no pressuposto de que as emoções, comportamentos e reações fisiológicas estão diretamente ligados à forma como o indivíduo avalia suas experiências no mundo (BECK; RUSH; SHAW; EMERY, 1997), ou seja, nessa perspectiva a visão e assimilação do individuo a respeito da situação torna-se mais importante do que o acontecimento por si só.

Logo, segundo Beck (1997), no modelo cognitivo, é possível identificar três níveis de cognição: os pensamentos automáticos, sendo nível mais superficial e espontâneo que surge na mente diante de diversas situações do cotidiano; as crenças intermediárias, que reflete a aparição de conteúdos cognitivos sob a forma de regras e suposições ligadas ao último nível e o mais profundo, que são as crenças centrais a respeito de si mesmo, dos outros e do mundo, que se formam a partir de experiências remotas da infância.

Ressalta-se a importância da postura empática e de acolhimento por parte do terapeuta, assim, por meio do estabelecimento desta relação, busca-se a colaboração e participação ativa do cliente e do terapeuta na resolução dos problemas abordados através de metas voltadas para o aqui e agora e para a identificação, avaliação e modificação de pensamentos e crenças disfuncionais. Para atingir tais objetivos, são utilizadas técnicas cognitivo comportamentais, principalmente o registro de pensamentos, o questionamento socrático e os experimentos comportamentais (ARAÚJO; SHINOHARA, 2002). É importante ressaltar que os terapeutas cognitivo-comportamentais também reconhecem que há interações complexas entre processos biológicos, influências ambientais, interpessoais, além de elementos cognitivos e comportamentais na gênese e no tratamento de transtornos psiquiátricos (WHRIGH; BASCO; THASE; 2008). 
A terapia cognitivo-comportamental (TCC) para os transtornos alimentares (TA) desenvolveu-se a partir de uma análise sistemática das perturbações emocionais, cognitivas e comportamentais características. Os programas de tratamento baseiam-se, amplamente, nas técnicas básicas para a redução da ansiedade, automanejo do comportamento e modificações de cognições mal adaptativas (CHANNON; WARDLE, 1994).

No momento presente, um dos principais representantes do modelo cognitivocomportamental para os transtornos alimentares é Fairburn. Sua abordagem relaciona pensamento, emoção e comportamento manifesto, e o tratamento tem como objetivo fazer com que o paciente examine a validade de suas crenças no presente e mude comportamentos disfuncionais. Os processos cognitivos mais frequentes nesses quadros (abstração seletiva, supergeneralização, magnificação, pensamento dicotômico, personalização e pensamento supersticioso) são definidos e examinados cuidadosamente, a fim de modificar os pensamentos e pressupostos automáticos (OLIVEIRA; DEIRO, 2013).

\section{Estratégias de tratamento para Transtornos Alimentares}

Dentre as principais estratégias utilizadas na mediação dos casos de TA segundo Duchesne e Almeida (2002), tem-se a Diminuição da restrição alimentar, relacionado ao estabelecimento de horários regulares para alimentação e na exposição gradual aos alimentos e situações frequentemente evitadas (GARNER; BEMI, 1985). Além disso, a TCC ensina técnicas de autocontrole ao paciente para redução de ansiedade, tristeza e outros sentimentos considerados facilitadores para a compulsão alimentar e a indução do vômito. (DUCHESNE; APPOLINÁRIO, 2001) Eliminação do uso de laxantes e diuréticos, feita de forma gradual. Diminuição da frequência de atividade física nos casos de anorexia e Aumento da atividade fisica nos casos de bulimia. Considerando também a Abordagem do distúrbio da imagem corporal, sendo esse ponto fundamental para o entendimento da crença central do caso. Para diminuir a distorção da percepção corporal pode-se solicitar à paciente que desenhe como percebe seu corpo, olhando-se em um espelho. Em seguida, o terapeuta desenha a silhueta real, para que a paciente possa observar a discrepância entre os desenhos. Modificação do sistema de crenças, os pacientes com transtornos alimentares apresentam crenças distorcidas e disfuncionais acerca de peso, formato corporal, alimentação e valor pessoal, que são significativas para a manutenção dos TA. Uma das crenças distorcidas centrais é a que equaciona valor pessoal ao peso e formato corporal, ignorando ou não valorizando outros parâmetros. (DUCHESNE; ALMEIDA, 2002) Para pacientes com TA, a magreza estaria 
associada à competência, superioridade e sucesso, tornando-se assim intrinsecamente associada à autoestima (GARNER; BEMIS, 1985).

Assim, são empregadas técnicas como registro de pensamentos, análise de evidências e a construção de pensamentos alternativos. É importante frisar que relacionado aos episódios de compulsão alimentar está o pensamento "tudo ou nada", que consiste em pensar em termos absolutos e extremos. Assim, pacientes com bulimia, por exemplo, adotam regras dietéticas inflexíveis e pequenos lapsos na dieta favorecem o abandono total do controle sobre a alimentação mudança (DUCHESNE; ALMEIDA, 2002), logo, ao invés de terem o pensamento de continuação do progresso, pensam que devem recomeçar e não levam em conta o degrau que já alcançaram.

A Abordagem da autoestima faz-se marcante devido ao processo de desmistificação e percepção de que esse fator pode e deve estar relacionado a outros aspectos, não somente relacionados à aparência física; ademais, quando associado à obesidade e excessiva atenção ao formato corporal, acompanha de sentimentos de vergonha e inferioridade, como ocorre no transtorno de compulsão alimentar (TCAP), em que há grande atenção aos esteriótipos sociais (DUCHESNE; ALMEIDA, 2002).

E por fim, é necessária a Avaliação da eficácia (DUCHESNE; ALMEIDA, 2002), que o terapeuta e paciente elencam as potencialidades e dificuldades do processo e da atual situação, devendo ser utilizadas técnicas para prevenção da recaída, que consistem em paciente e terapeuta identificarem possíveis dificuldades futuras e planejarem estratégias adequadas para lidar com essas mudança. Ressalta-se a expressividade para o processo terapêutico, a posição realista em relação à meta de peso, modificando as crenças relacionadas ao peso e ao formato corporal, alcançando um equilíbrio entre autoaceitação e mudança (DUCHESNE; ALMEIDA, 2002).

É relevante analisar os grupos de gatilhos que podem desencadear um episódio de compulsão alimentar, para que seja possível trabalhar com quais sejam o caso do paciente, podendo ser: Biológico, como o vazio no estômago ou desejos influenciados pelos hormônios; Ambientais, visão de alimentos seja presencial ou virtual e o cheiro; Mentais, que são regidos pelos pensamentos ou lembranças sobre comida; Emocionais, emoções negativas ou positivas associadas à vontade de comer, seja para suprir as negativas ou comemorar as positivas; Sociais, incentivado a partir de outras pessoas comendo (BECK, 2009).

Elencar objetivos realistas do processo terapêutico é primordial, também pelo fato de serem essenciais na manutenção da motivação. Para isso, é interessante escrever uma espécie de cartão de motivação, que são as razões pelas quais o paciente deseja solucionar a queixa 
inicial. Em outro momento, podem ser escitas respostas adaptativas aos pensamentos dissociativos, ao pensamento "tudo ou nada" citado anteriormente. Exemplo: ontem tive um lapso, na semana que vem recomeço do zero o processo; pode ser substituído por: ontem tive um lapso, o que sabia que poderia acontecer, entretanto, continuarei o processo de onde eu parei pois já detive algum aprendizado (BECK, 2009).

A psicoeducação deve fazer parte do processo havendo o aprendizado da análise dos erros cognitivos, que são grupos quais os pensamentos disfuncionais se encaixam. Whrigh, Basco e Thase (2008, grifo nosso) apresentam alguns deles:

Abstração seletiva, podendo ser chamado de ignorar as evidências ou filtro mental, afim de confirmar a visão que a pessoa tem da situação; Inferência Arbitrária chega-se a uma conclusão a partir de evidências contraditórias ou ausência de evidências; Supergeneralização, a conclusão de um acontecimento isolado ou de determinada área da vida da pessoa é estendida de maneira ilógica para as demais áreas do funcionamento; Maximização, a relevância de um acontecimento ou atributo é exagerada ou minimizada no caso da Minimização; Personalização, assume-se responsabilidade excessiva ou culpa por eventos negativos, enquanto na realidade não tem relação a si próprio; Pensamento Absolutista, também chamado de dicotômico ou do tipo "tudo ou nada", em que os julgamentos sobre si e experiências ou são totalmente boas ou totalmente ruins, fracasso ou perfeição.

Coeso a isso, Whrigh, Basco e Thase (2008) defendem a Técnica das Sete Perguntas:

1. Que tipo de erro estou cometendo? 2. Qual a evidência de que esse pensamento não seja verdadeiro (totalmente ou parcialmente)? 3. Existe uma outra maneira de ver a situação? 4. Qual o resultado mais realista para a situação? 5. Qual o efeito de acreditar nesse pensamento e qual seria de acreditar em um pensamento alternativo? 6. O que eu diria para [um amigo] se eles estivessem na mesma situação com os mesmos pensamentos? 7. O que eu devo fazer agora? De acordo com tais exemplos de pensamentos citados, é possivel perceber que a psicoeducação é fundamental para a reestruturação cognitiva, ou seja, para mudança de pensamento de modo que interfira nos comportamentos desadaptativos do indivíduo.

Além disso, aprender a se planejar é fundamental, podendo escrever as tarefas que deseja realizar em um bloco de notas ou caderno, de forma que seguir a rotina escrita pode se tornar mais fácil, se comportar evitando o excesso de comida fora do planejado ou agir seguindo a alimentação das comidas planejadas anteriormente. Momentos de elogio (BECK, 2009) também são valorosos como uma autoafirmação positiva, sendo importante elaborar essa habilidade caso não haja, visto que, pensamentos e palavras reforçadoras são necessárias para a percepção do próprio paciente sobre o andamento do processo e o crescimento de suas competências. 


\section{Desenvolvimento do Jogo}

O jogo Nova Luz se apresenta em forma de tabuleiro retangular sob uma temática de viagem de trem, tem como proposta a utilização em sessão de terapia sobretudo na abordagem cognitivo comportamental e em casos de transtornos alimentares. O jogo se inicia em uma estação de trem, onde como primeiro passo o paciente deve montar seu personagem com partes do corpo disponíveis, podendo assinalar quais partes o incomoda, assim já é possível averiguar a maneira como este se enxerga e tem a concepção de si, podendo identificar possíveis distorções; e tem como o final a saída de um túnel que irradia luz e o desembarque a estação de destino. O jogo apesar de lúdico é indicado para adolescentes e adultos, tem o intuito de tratar a teoria e ensinamentos de maneira descontraída e que as respostas do paciente se deem de forma espontânea, assim como a averiguação do caso.

Existe uma brincadeira com as palavras na construção do material, no título e ao desenrolar do jogo, Nova Luz remete à "luz no fim do túnel”, por isso a temática ferroviária com passagens por túneis e o trem como meio de locomoção, que é responsável por transportar o paciente para tais situações, perguntas, ensinamentos e simulações de tarefas cotidianas. Investindo na temática lúdica, Nova Luz conta com personagens secundários conhecidos como o gênio da lâmpada e os macaquinhos dos 3 sentidos, além de desafios impostos na jogada. O locutor do jogo é o maquinista, sendo interpretado pelo psicólogo em questão e a peça utilizada para locomoção é um vagão de trem que se encarregará de levar também as respostas escritas pelo paciente durante o jogo.

O material conta com momentos de análise da demanda inicial e das motivações pessoais. Havendo momentos de psicoeducação como a explicação de sigilo e ética profissional, da TCC, do modelo cognitivo, dos erros cognitivos, questionamento de evidências, pensamentos desadaptativos e adaptativos, além da confecção de um cartão de enfrentamento. Para manutenção da autoestima, há momentos de ensinamento do que significa atrelado à situação em que se encontra no jogo, enumeração das do paciente habilidade e da prática de se elogiar. Também dispõe de situações indagando se na rotina do paciente há presença de dieta, exercício fisico, equipe multidisciplinar e apoio pessoal.

No caminho representado pela linha do trem existem círculos coloridos que correspondem às paradas: o roxo é primeiro círculo orientando que o avatar deve ser montado, vermelho quando for simplesmente para responder em voz alta, azul com o número condizente da carta que deve ser retirada, verde com o símbolo de lápis expondo que é para escrever a resposta e depositá-la no vagão. O personagem referente a fala é indicado em cada 
balão. Já as estrelas amarelas ilustradas no decorrer do tabuleiro são as curiosidades no estilo "Você sabia?", na qual sempre que passar o paciente deve escolher livremente uma carta em forma de estrela e entregar ao psicólogo para que leia em voz alta, dentre essas curiosidades está: a importância de se elogiar; o tempo que leva para perceber que está realmente saciado; a diferença da fome, vontade e desejo de comer; os diferentes estímulos que levam a comer; as orientações de como comer consciente; o ensinamento da respiração; a explicação de que o processo de cura não é linear; e a técnica A.C.A.L.M.E-S.E idealizada por Bernad Rangé, defendendo a ideia de que a melhor maneira de lidar com o estado de ansiedade é aceitando-o, para isso a técnica se divide em 8 passos: Aceite a sua ansiedade; Contemple as coisas em sua volta; Aja com sua ansiedade; Libere o ar de seus pulmões; Mantenha os passos anteriores; Examine seus pensamentos; Sorria, você conseguiu; Espere o futuro com aceitação (RANGÉ, 2001).

Portanto, este jogo tem como intuito auxiliar em casos relacionados aos transtornos alimentares, ao abordar a queixa inicial e as motivações do paciente, a versar sobre a autoestima, a consciência alimentar e como lidar a respeito de comportamentos ligados à ansiedade, além de explicar como funciona a abordagem cognitivo comportamental. Pode ser usado logo ao início do processo terapêutico para contribuir ao tratar de questões já citadas relacionadas ao transtorno alimentar ou em momentos mais a frente em psicoterapia como um utensílio de manutenção e análise do andamento do caso, assim como a ratificação dos ensinamentos.

\section{Considerações finais}

O presente estudo buscou expor a contextualição das questões alimentares atuais, ressaltando os rodízios, os fast-food, a fartura e a valorização da comida, em contrapartida do enaltecimento do corpo magro perfeito evidenciado pelas mídias, sobretudo fruto das redes sociais. Além disso, foram citados dados mostrando a diminuição da desnutrição e aumento da obesidade encontrados na população brasileira.

Em relação aos transtornos alimentares existentes, foram abordados principalmente a compulsão alimentar, anorexia e bulimia, visto que são os que se apresentam de forma mais específica na literatura, além da correlação a respeito da preocupação corporal e pressão social. Sendo assim, foram argumentados o histórico e as condições diagnósticas, seguidos pela revelação de fatores multifatoriais, uma vez que existe uma diversidade de fatores que interagem entre si de modo complexo para produzir, desenvolver e perpetuar a doença, sendo 
estes compostos de predisposições genéticas, socioculturais e vulnerabilidades biológicas e psicológicas.

A respeito da terapia cognitivo-comportamental, foi apresentado um breve histórico e caracterização, sendo esta uma abordagem que se baseia no modelo cognitivo e utiliza técnicas e estratégias comportamentais, desse modo, foram apresentadas estratégias utilizadas por psicoterapeutas em casos de transtornos alimentares, resultando na contribuição de ideias relacionadas ao desenvolvimento do jogo.

Concluiu-se possível a elaboração do jogo com o intuito de auxiliar em casos relacionados aos transtornos alimentares, de modo que a sessão se torne menos tensa e mais fluida, além da concretização da ideia que embora houvesse a utilização do recurso lúdico, que este fosse utilizado para a psicoterapia com adolescentes ou adultos, logo, que não convertesse à um material infantilizado. Pode-se firmar a opção da utilização logo ao início do processo terapêutico, para contribuir ao tratar de questões já citadas relacionadas ao transtorno alimentar, como também em momentos mais a frente em psicoterapia como um utensílio de manutenção e análise do andamento do caso, assim como a ratificação dos ensinamentos já apreendidos durante o jogo.

O Brasil ainda não dispõe de grande quantidade de material com a temática dos transtornos alimentares, com isso, entende-se que é uma área em progresso que necessita de estudos. Já os jogos, atualmente utilizados como recursos em psicoterapia, em sua maneira geral, são direcionados ao atendimento infantil. Assim, esse estudo une dois fatores ainda em avanço na terapia cognitivo-comportamental brasileira.

\section{REFERÊNCIAS}

AFFONSO, R. M. L. (Org.). Ludodiagnóstico: investigação clinica através do brinquedo. Porto Alegre: Artmed, 2012. p. 266.

AMERICAN PSYCHIATRIC ASSOCIATION. Manual Diagnóstico e Estatístico de Transtornos Mentais: DSM-5. Porto Alegre: Artmed, 2014.

ARAÚJO, C. F.; SHINOHARA, H. Avaliação e diagnóstico em terapia cognitivocomportamental. Interação em Psicologia, Curitiba, v. 6, n 1, p. 37-43, jan./jun. 2002.

BECK, A. T.; RUSH, A. J.; SHAW, B. F.; EMERY, G. Terapia cognitiva da depressão. Porto Alegre: Artes Médicas, 1997.

BECK, J. S. Pense magro: a dieta definitiva de Beck. Porto Alegre: Artmed, 2009. 317 p. 
BORGES, N. J. B. G. et al. Transtornos alimentares: quadro clínico. Revista da Faculdade de Medicina de Ribeirão Preto, Ribeirão Preto, v. 39, n. 3, p. 340-348, jul./set. 2006.

CHANNON, S.; WARDLE, J. Transtornos alimentares. In: SCOTT, J. et al. (Eds.). Terapia cognitiva na prática clínica. Porto Alegre: Artes Médicas, 1994.

CORDÁS, T. A. Transtornos alimentares: classificação e diagnóstico. Revista Psiquiatria Clinica, São Paulo, v. 31, n. 4; p. 154-157, 2004.

CORDÁS, T. T.; CLAUDINO, A. M. Transtornos alimentares: fundamentos historicos. Revista Brasileira de Psiquiatria, São Paulo, v. 24, supl. 3, p. 3-6, dez. 2002.

DUCHESNE, M.; ALMEIDA, P. E. M. Terapia cognitivocomportamental dos transtornos alimentares. Revista Brasileira de Psiquiatria, São Paulo, v. 24, supl. 3, p. 49-53, dez. 2002.

DUCHESNE, M.; APPOLINÁRIO, J.C. Tratamento dos transtornos alimentares. In:

RANGÉ, B. (Ed.). Psicoterapias cognitivocomportamentais: um diálogo com a psiquiatria. São Paulo: Artmed, 2001. p. 317-31.

FARIA, P.; SHINOHARA, H. Transtornos Alimentares. Revista Interação, Curitiba, v. 2, n. 2, p. 51-73, 1998.

GARNER, D. M.; BEMIS, K. M. Cognitive therapy for anorexia nervosa. In: GARNER D. M.; GARFINKEL P. E. (Eds.). Handbook of psychotherapy for anorexia nervosa e bulimia. New York: The Guilford Press, 1985. p. 46-107.

LEAL, C. A.; CABRAL, M. D. Complicações clínicas da anorexia nervosa e bulimia nervosa. Rio de Janeiro, 2002.

OLIVEIRA, L. L. Padrões disfuncionais de interação em famílias de adolescentes com anorexia nervosa. 2004. 149 f. Dissertação (Mestrado em Psicologia) - Universidade Federal do Rio Grande do Sul, Porto Alegre, 2004.

OLIVEIRA, L. L.; DEIRO, C. P. Terapia Cognitivo-Comportamental para Transtornos Alimentares: a visão de psicoterapeutas sobre o tratamento. Revista Brasileira de Terapia Comportamental e Cognitiva, São Paulo, v. 14, n. 1, p 36-49, 2013.

OLIVEIRA, L. L.; HUTZ, C. S. Transtornos Alimentares: O papel dos aspectos culturais no mundo contemporâneo. Revista Psicologia em Estudo, Maringá, v. 15, n. 3, p. 575-582, jun./set. 2010.

RANGÉ, B. Vencendo o pânico: instruções passo-a-passo para quem sofre de ataques de pânico. Rio de Janeiro: Instituto de Psicologia, Universidade Federal do Rio de Janeiro, 2001.

WHRIGH, J. H.; BASCO, M. R.; THASE, M. E. Aprendendo a terapia cognitivocomportamental: um guia ilustrado. Porto Alegre: Artmed, 2008. 


\section{Como referenciar este artigo}

SARTORATO, R.; RIBEIRO, L. P. Desenvolvimento do jogo Nova Luz para utilização na psicoterapia em casos de transtornos alimentares. Doxa: Rev. Bras. Psico. e Educ., Araraquara, v. 22, n. esp. 1, p. 347-361, out., 2020. e-ISSN: 2594-8385. DOI: https://doi.org/10.30715/doxa.v22iesp.1.14138

Submetido em: 20/04/2020

Revisões requeridas: $15 / 06 / 2020$

Aprovado em: 20/08/2020

Publicado em: 30/09/2020 